\title{
A NOVEL ELECTROSTATICALLY ACTUATED AFM PROBE FOR VIBRO- FLEXURAL MODE OPERATION
}

\author{
E. Sarajlic ${ }^{1}$, M.H. Siekman ${ }^{2}$, H. Fujita ${ }^{3}$, L. Abelmann ${ }^{2}$ and N. Tas ${ }^{2}$ \\ ${ }^{1}$ SmartTip B.V., Enschede, THE NETHERLANDS \\ ${ }^{2}$ MESA+, University of Twente, Enschede, THE NETHERLANDS \\ ${ }^{3}$ CIRMM, Institute of Industrial Science, The University of Tokyo, Tokyo, JAPAN
}

\begin{abstract}
A successful approach to drastically reduce or even completely eliminate friction and wear in scanning force microscopy is the use of electrostatic modulation of the normal force acting on the tip-sample contact. In this paper we have devised, fabricated and experimentally characterized a novel electrostatically actuated AFM probe. The probe consists of a flexible cantilever that has an electrostatic circular plate actuator with a built-in sharp tip monolithically integrated at its free end. This unique probe configuration will allow for the vibro-flexural mode operation in which vibration of the tip relative to the cantilever is generated and controlled by the integrated plate actuator, while the tip-sample interaction is resolved by deflection of the cantilever. We envision that this new operation mode will result in an efficient electrostatic force modulation, which in the end will enable us to control friction and wear during AFM imaging.
\end{abstract}

\section{INTRODUCTION}

Atomic force microscopy (AFM) in a high resolution tapping mode can be performed by applying electrostatic force modulation on the AFM cantilever instead of a conventional mechanical vibration of the entire probe [1]. More importantly, electrostatic modulation of the normal tip-sample force can be used to drastically reduce or even completely eliminate friction and wear in scanning force microscopy $[2,3]$.

In general, electrostatic force modulation is performed using standard AFM cantilever-type probes. In this paper, we propose a new electrostatically actuated AFM probe, which will allow for a more efficient force modulation and therefore better control of friction and wear during surface imaging.

\section{WORKING PRINCIPLE}

Both conventional and novel electrostatically actuated AFM probes are schematically illustrated in Figure 1.

A conventional probe consists of a cantilever with a sharp tip at its end. The cantilever is usually made of a conductive material or a dielectric material coated with a metallic layer. Normal vibration of the cantilever can be induced by applying an oscillating voltage between the cantilever and the conductive substrate. By exciting the cantilever on resonance during AFM imaging an effective elimination of friction, so called superlubricity, can be achieved $[2,3]$. Because the electrostatic force is generated between the cantilever and the sample, a practical

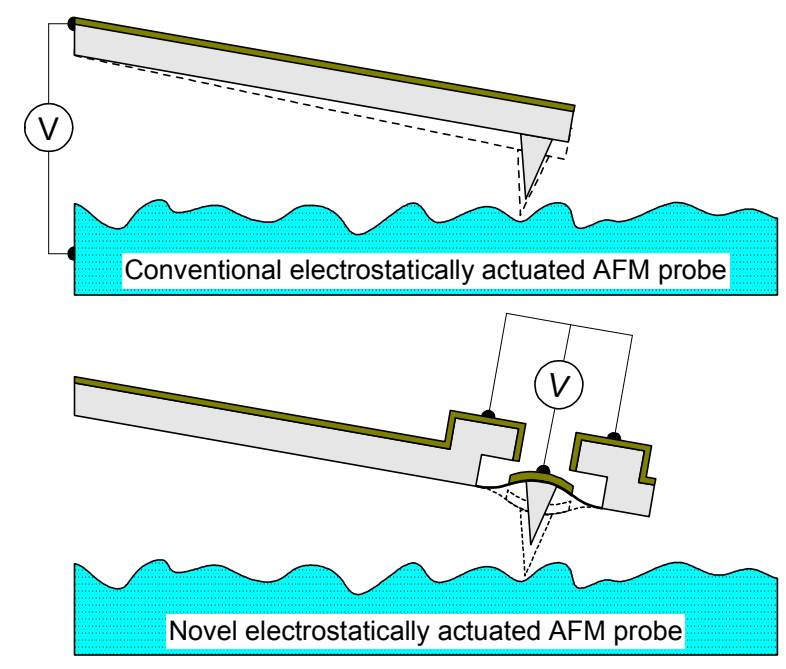

Figure 1: Electrostatically actuated AFM probes: conventional (above) and novel active probe (below).

application of conventional probes is limited to surface imaging of the conductive substrates. Furthermore, the magnitude of the electrostatic force depends on various geometric parameters such us the tip height, the angle between the cantilever and the sample, the possible stressinduced deformation of the cantilever and the topography of the sample surface. These uncertain parameters make the precise control of the electrostatic force and therefore a stable small-amplitude oscillation of the cantilever, which is essential for reduced friction and wear, rather challenging.

On the other hand, the novel probe has an electrostatic plate actuator monolithically integrated at the end of the cantilever. The electrostatic actuator consists of an elastic circular plate with a sharp tip located at its center and a relatively rigid counter electrode. When a potential difference is applied between the plate and the counter electrode an electrostatic force is generated. The generated force causes deflection of the plate moving the probe tip relative to the cantilever. This unique probe configuration will allow for the vibro-flexural mode operation. In this operation mode vertical vibration of the tip can be generated and controlled by the integrated plate actuator. At the same time the tip-sample interaction can be resolved by deflection of the cantilever. Because electrostatic actuation of the plate actuator is completely independent from the substrate, the new probe will enable scanning of any surface regardless of its electrical conductivity. Furthermore, the well-defined geometry and dimensions of the plate actuator will ensure a 
reliable electrostatic force generation and consequently allow a more stable small-amplitude oscillation of the probe tip. Finally, the high-bandwidth plate actuator will allow a more efficient excitation of the normal tip-sample force.

\section{MICROFABRICATION PROCESS}

The probe is realized in a wafer-scale fabrication process by conventional surface and bulk micromachining techniques. The relatively simple fabrication process, schematically illustrated in Figure 2, employs only three photolithographic masks.
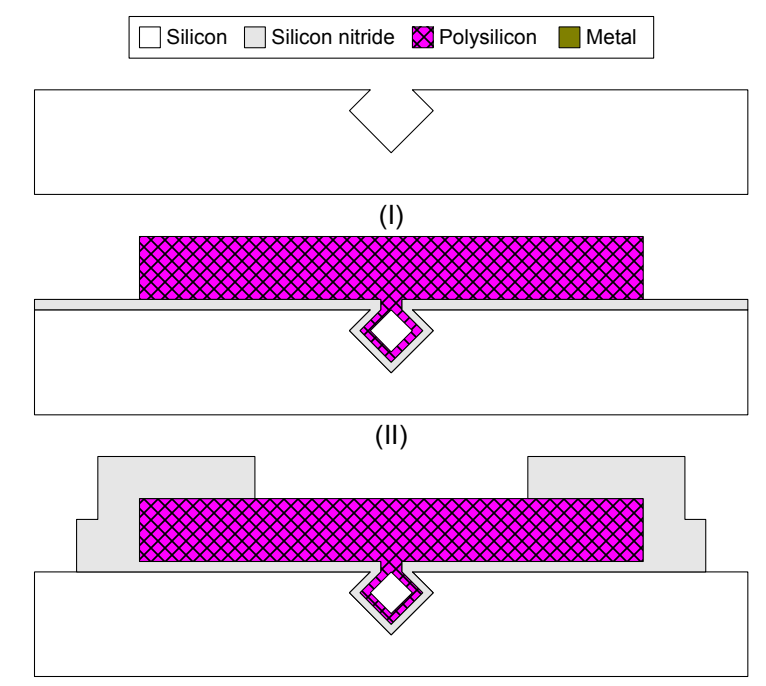

(III)

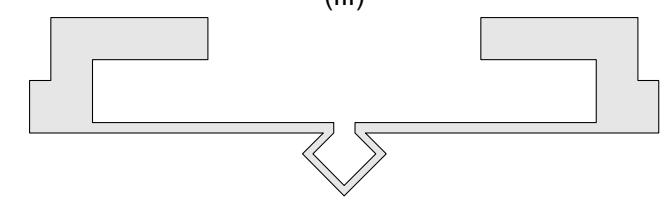

(IV)

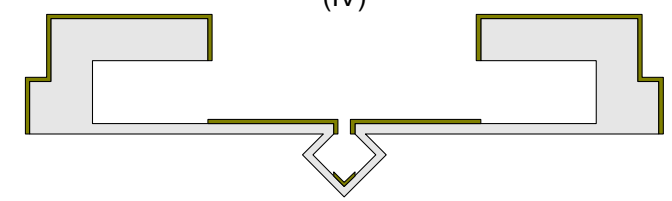

(V)

Figure 2: The process of wafer-scale fabrication of the novel AFM probe using standard surface and bulk micromachining techniques.

The process starts on a (100)-oriented standard silicon wafer. In the selected wafer a silicon cavity is formed by a combination of deep reactive ion etching (DRIE) and wet anisotropic etching in $\mathrm{KOH}$ (Step I) [4]. The formed octahedron cavity bounded by $<111>$ crystallographic planes serves as a mold for a sharp probe tip with a relatively large height. After formation of the mold, we deposit a first silicon nitride layer by Low Pressure Chemical Vapor Deposition (LPCVD). In the next fabrication step, an LPCVD polysilicon layer is deposited and patterned by RIE (Step II). Polysilicon serves both as the sacrificial and the structural material. After the

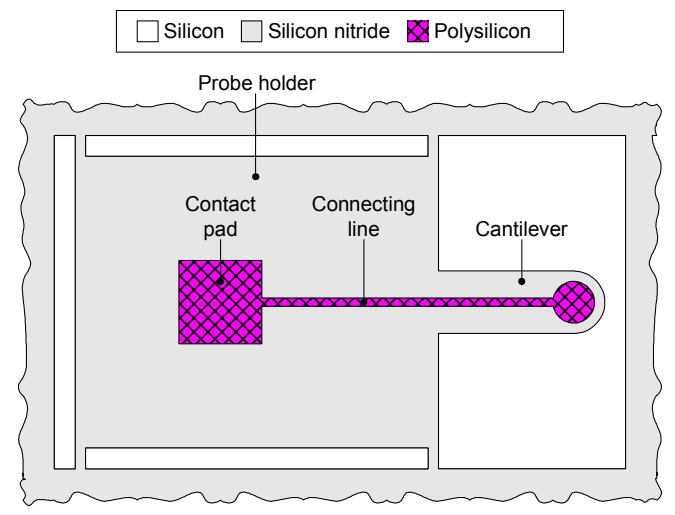

Figure 3: The layout of the probe prior to the release by wet anisotropic etching in a hot TMAH solution. .

patterning of polysilicon, a second LPCVD silicon nitride layer is deposited. Subsequently, silicon nitride is patterned by RIE to define the probe layout and to open the etch holes in the top silicon nitride layer that give access to the sacrificial parts of the polysilicon layer (Step III). The layout of the probe and the etch holes are depicted in Figure 3. In the next fabrication step, the substrate is placed in a hot TMAH solution in order to partially dissolve bulk silicon and to completely remove sacrificial polysilicon (Step IV). Etching of the bulk silicon proceeds from both front and back side of the substrate. The silicon nitride mask, shown in Figure 3, guides the front side etching process allowing complete removal of silicon underneath the cantilever while protecting the bulk material below the prospective probe holder. At the same time, the substrate is etched from the back side in order to release the entire probe and to adjust the probe holder to the desired final thickness. The final thickness of the probe holder has to be roughly six times smaller than the cantilever length in order to ensure a proper contact of the probe tip during AFM imaging. In the same processing step, sacrificial polysilicon is removed in order to release the plate and to define the contact pad and the connecting electric line between the pad and the plate. Part of the polysilicon layer, which is fully encapsulated by silicon nitride, remains protected during the sacrificial etching and is used as the structural material for the

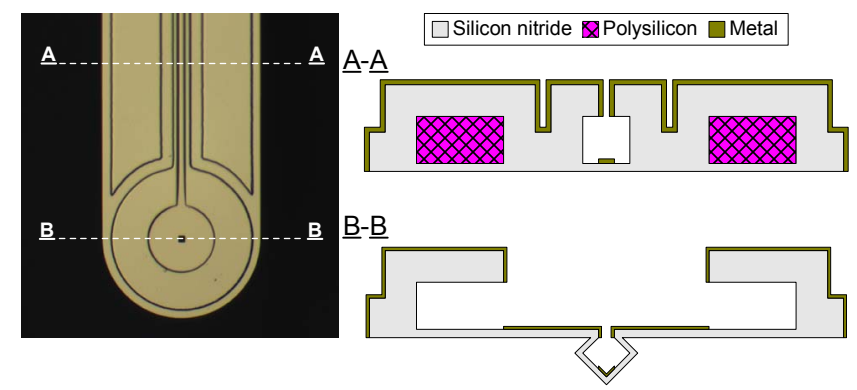

Figure 4: Different cross-sections of the probe cantilever. The undercut of the top silicon nitride layer ensures proper electrical insulation between the upper and the lower electrodes. The encapsulated polysilicon layer provides the mechanical strength and rigidity of the cantilever. 

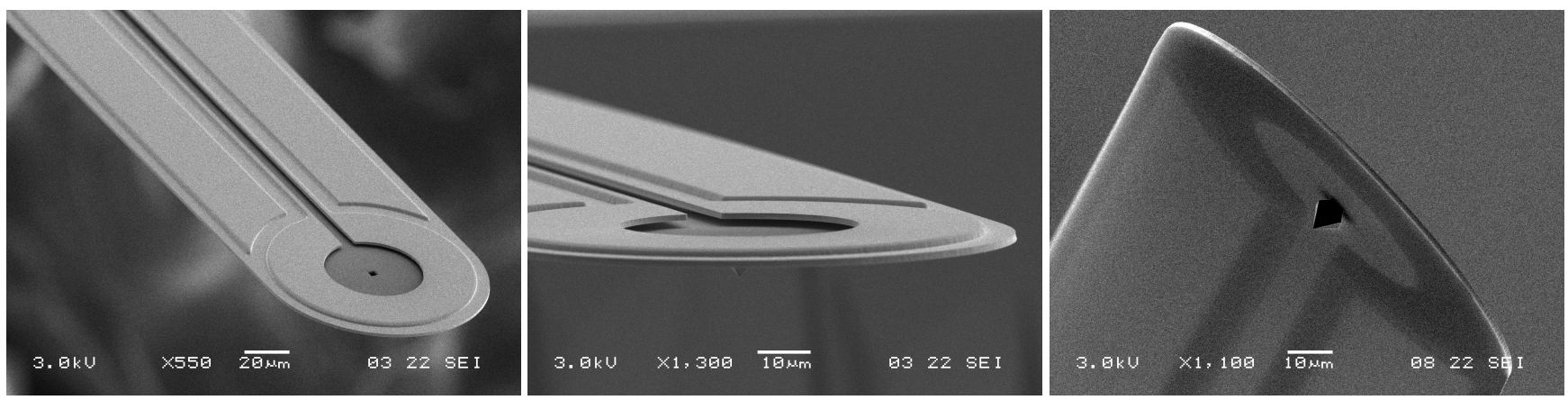

Figure 5: SEM micrographs of the electrostatically actuated AFM probe. The probe consists of an electrostatic circular plate actuator with a built-in sharp tip monolithically integrated at the end of an AFM-type cantilever. The cantilever is 550 $\mu \mathrm{m}$ long, $110 \mu \mathrm{m}$ wide and has an aggregated thickness of $2.55 \mu \mathrm{m}\left(200 \mathrm{~nm} \mathrm{Si}_{R} N / 1.5 \mu \mathrm{m}\right.$ polysilicon/800 $\mathrm{nm} \mathrm{Si} i_{R} N / 50 \mathrm{~nm}$ $\mathrm{Au}$ ). The diameter and the thickness of the plate are $100 \mu \mathrm{m}$ and $200 \mathrm{~nm}$, respectively. The gap between the plate and the counter electrode is $1.5 \mu \mathrm{m}$.

cantilever, as illustrated in Figure 4. The upper and the lower electrodes of the electrostatic circular plate actuator are formed by directional sputter deposition of a thin metal coating (Step V). The undercut of the top silicon nitride layer ensures proper electrical insulation between the lower and the upper electrodes.

\section{MICROFABRICATION RESULTS}

SEM micrographs of the successfully fabricated probe are shown in Figure 5. The probe cantilever is $550 \mu \mathrm{m}$ long and $110 \mu \mathrm{m}$ wide. The cantilever is made of three structural layers: (i) $200 \mathrm{~nm}$ thick silicon nitride layer at the bottom, (ii) $1.5 \mu \mathrm{m}$ thick polysilicon layer in the middle and (iii) 800 $\mathrm{nm}$ thick silicon nitride layer on the top. At the end of the cantilever a circular plate actuator is monolithically integrated. The actuator plate has a diameter of $100 \mu \mathrm{m}$. At the center of the plate a sharp probe tip is placed. The height of the tip is approximately $10 \mu \mathrm{m}$. The plate is realized in the bottom silicon nitride layer and therefore has a thickness of $200 \mathrm{~nm}$. On the other hand the counter electrode is defined in the top silicon nitride layer and has a corresponding thickness of $800 \mathrm{~nm}$. The gap between the plate and the counter electrode, which is defined by removal of the sacrificial polysilicon layer, is $1.5 \mu \mathrm{m}$. The probe cantilever is metallized by a $50 \mathrm{~nm}$ thick gold layer in order to define electrodes.

\section{EXPERIMENTAL RESULTS}

After fabrication, we have first confirmed a proper electrical insulation of the probe by measuring the electrical resistance between the lower and the upper electrodes of the electrostatic plate actuator. Subsequently, the probe is experimentally characterized by Scanning Laser-Doppler Vibrometery using a Polytec Micro System Analyzer MSA400. A grid of 230 measurement points uniformly distributed over the probe and a part of the probe holder is used. The probe is excited by applying an ac actuation voltage with a $4 \mathrm{~V}$ amplitude and a $4 \mathrm{~V}$ dc component on the plate actuator. Figure 6 shows the first six vibration modes of the cantilever. The first, second and fourth modes are normal bending modes, while the third mode is a torsional mode. The fifth and sixth modes are complex higher-order modes of vibration. The fundamental vibration mode of the circular plate is found to be $530 \mathrm{kHz}$. Frequency spectrums for two measurement points, respectively at the end of the cantilever and at the center of the plate, are shown in Figure 7. From the frequency graph we can clearly see that the vibration of the cantilever is
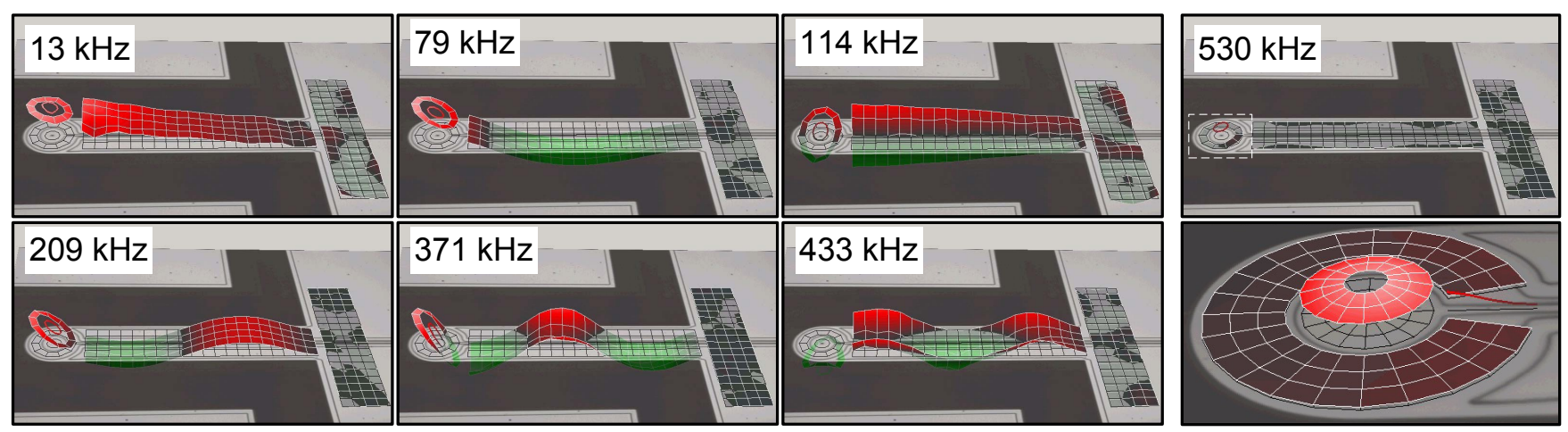

Figure 6: Out-of-plane vibration of the probe is measured by Scanning Laser-Doppler Vibrometry using the Polytec Micro System Analyzer MSA-400. (left) The first six vibration modes of the cantilever; (right) The first vibration mode of the circular plate. The probe is excited by applying a driving voltage of $4 \mathrm{~V}$ ac plus $4 \mathrm{~V} d \mathrm{c}$ at the electrostatic plate actuator. 
efficiently excited only when the electrostatic plate actuator is driven around the resonance frequencies of the cantilever.

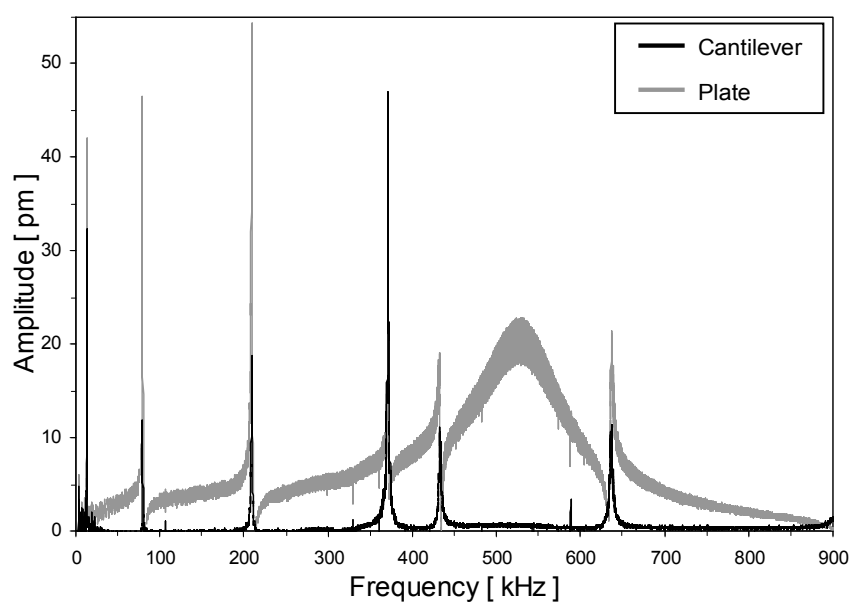

Figure 7: Measured frequency spectrum of the cantilever and the plate. Data were taken at an ac driving voltage with a $4 \mathrm{~V}$ amplitude and a $4 \mathrm{~V}$ dc component.

Finally, we have measured the mid-point deflection of the circular plate as a function of the actuation voltage. In general, the electrostatic force is proportional to the square of the applied voltage. When an electrostatic actuator is driven by an alternating voltage at a certain frequency, the generated force has a double frequency component [1]. Therefore we have actuated the plate actuator using a sinusoidal voltage signal at a frequency of $75 \mathrm{kHz}$ and measured the plate response at a frequency of $150 \mathrm{kHz}$. Measurement data are shown in Figure 8.

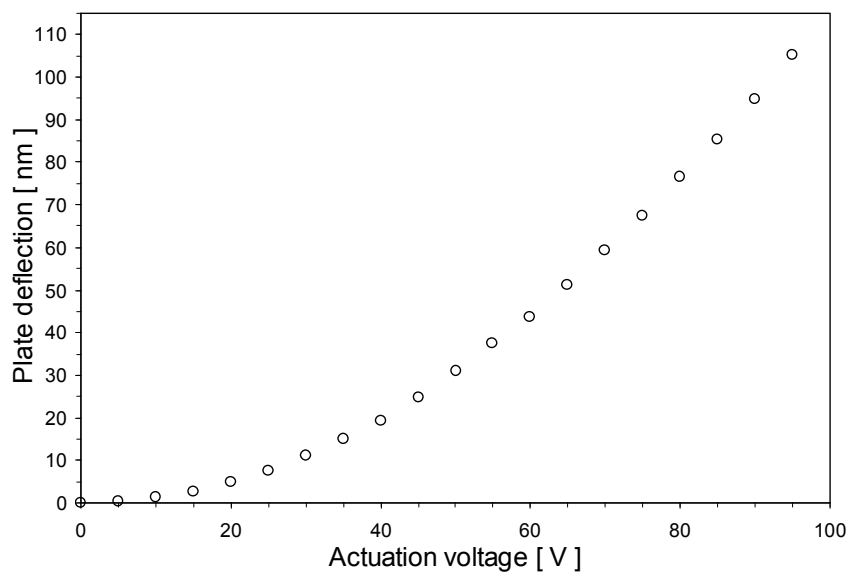

Figure 8: Measured mid-point deflection of the plate versus actuation voltage. Data were taken at a frequency of 150 $k H z$.

From the graph we can confirm quadratic dependence of the deflection with the driving voltage, as expected for electrostatic actuation. The maximum mid-point deflection of $105 \mathrm{~nm}$ is measured using an actuation voltage of $95 \mathrm{~V}$.

\section{CONCLUSION AND OUTLOOK}

We have developed a novel AFM cantilever probe with an electrostatic circular plate actuator monolithically integrated at its free end. The probe with this unique configuration will enable new scanning mode operation that will likely enable us to switch friction on and off during AFM imaging. Furthermore, the high-bandwidth plate actuator with an integrated sharp tip could be employed for high-speed scanning probe microscopy [5-7].

In our future research we would like to manufacture a suitable AFM holder with proper electrical connections in order to be able to test our probe in an atomic force microscope. Furthermore, we plan to derive appropriate electrical and mechanical models to improve our understanding of this type of probes.

\section{ACKNOWLEDGMENTS}

The authors would like to thank the staff of the MESA+ Clean Room for their technical assistance with probe fabrication. We are grateful to P. Linders from Deltamask for the masks processing. The authors acknowledge R.G.P. Sanders from the TST group for his valuable support during the experimental characterization of the probe.

\section{REFERENCES}

[1] J.W. Hong, Z.G. Khim, A.S. Hou, Sang-il Park, "Tapping mode atomic force microscopy using electrostatic force modulation", Appl. Phys. Lett., vol. 69(19), pp. 2831-2833, 1996.

[2] A. Socoliuc, E. Gnecco, S. Maier, O. Pfeiffer, A. Baratoff, R. Bennewitz, E. Meyer, "Atomic-scale control of friction by actuation of nanometer-sized contacts", Science, vol. 313, pp. 207-210, 2006.

[3] M.A. Lantz, D. Wiesmann, B. Gotsmann, "Dynamic superlubricity and the elimination of wear on the nanoscale", Nature Nanotechnology, vol. 4, pp. 586591, 2009.

[4] A. Boisen, J.P. Rasmussen, O. Hansen, S. Bouwstra, "Indirect tip fabrication for scanning probe microscopy", Microelectronic Engineering, vol. 30, pp. 579-582, 1996.

[5] F.L. Degertekin, A.G. Onaran, M. Balantekin, W. Lee, N.A. Hall, C.F. Quate, "Sensor for direct measurement of interaction forces in probe microscopy", Appl. Phys. Lett., vol. 87, pp. 213109, 2005.

[6] A.G. Onaran, M. Balantekin, W. Lee, W.L. Hughes, B. A. Buchine, R.O. Guldiken, Z. Parlak, C.F. Quate, F.L. Degertekin, "A new atomic force microscope probe with force sensing integrated readout and active tip", Rev. Sci. Instrum., vol. 77, 023501, 2006.

[7] E.C.M. Disseldorp, F.C. Tabak, A.J. Katan, M.B.S. Hesselberth, T.H. Oosterkamp, J.W.M. Frenken, W.M. van Spengen, "MEMS-based high speed scanning probe microscopy", Rev. Sci. Instrum., vol. 81, pp. 043702, 2010. 